EPJ Web of Conferences 73, 07004 (2014)

DOI: $10.1051 /$ epjconf/20147307004

(C) Owned by the authors, published by EDP Sciences, 2014

\title{
Test of fundamental symmetries via the Primakoff effect
}

\author{
Liping Gan ${ }^{\mathrm{a}, \mathrm{b}}$ \\ University of North Carolina Wilmington, NC, USA
}

\begin{abstract}
The three neutral pseudoscalar mesons, $\pi^{0}, \eta$ and $\eta^{\prime}$, represent one of the most interesting systems in strong interaction physics. A study of the electromagnetic properties of these mesons provides a sensitive probe of the symmetry structure of QCD at low energy. A comprehensive experimental program at Jefferson Laboratory (Jlab) is aimed at gathering high precision measurements on the two-photon decay widths and transition form factors at low $Q^{2}$ of $\pi^{0}, \eta$ and $\eta^{\prime}$ via the Primakoff effect. The completed experiments on the $\pi^{0}$ radiative decay width at Jlab $6 \mathrm{GeV}$, and other planned measurements at Jlab $12 \mathrm{GeV}$ will provide a rich laboratory to test the chiral anomaly and to study the origin and dynamics of chiral symmetry breaking at the confinement scale of QCD.
\end{abstract}

\section{Introduction}

The symmetries of QCD at low energy are manifested in their most unambiguous form in the sector of light pseudoscalar mesons $\pi^{0}, \eta$ and $\eta^{\prime}$. While $\pi^{0}$ and $\eta$ are Goldstone bosons due to spontaneous chiral symmetry breaking, the $\eta^{\prime}$ is not due to the $\mathrm{U}(1)_{A}$ anomaly. There is a second type of axial anomaly driving the two-photon decays of $\pi^{0}, \eta$ and $\eta^{\prime}$. This system harbors fundamental information about the effects of SU(3) symmetry and the mixing phenomena of the mesons due to isospin and SU(3) symmetry breaking. It opens a new window to determine the fundamental parameters of QCD in a modelindependent manner, such as the light quark-mass difference $m_{d}-m_{u}$ and the mixing angle of $\eta-\eta^{\prime}$.

The availability of a high duty factor continuous electron beam at Jlab offers a great opportunity to perform precision measurements. A comprehensive Primakoff experimental program has been developed by the PrimEx collaboration in the last decade at Jlab to measure the two photon decay widths, $\Gamma_{\gamma \gamma}$, and the transition form factors, $F_{\gamma \gamma^{*}}$, of $\pi^{0}, \eta$, and $\eta^{\prime}[1,2]$. The radiative decay width of $\pi^{0}$ has been measured by two experiments (PrimEx-I and PrimEx-II) at Jlab $6 \mathrm{GeV}$. The advent of Jlab $12 \mathrm{GeV}$ upgrade will extend the program to more massive $\eta$ and $\eta^{\prime}$. The transition form factors of these mesons in the low $Q^{2}\left(0.001-0.5 \mathrm{GeV}^{2} / \mathrm{c}^{2}\right)$ region will be measured at $12 \mathrm{GeV}$ as well.

\section{Neutral pion lifetime measurements at Jlab $6 \mathrm{GeV}$}

The two-photon decay of $\pi^{0}$ reveals one of the most profound symmetry issues in QCD, namely, the explicit breaking of a classical symmetry by the quantum fluctuations of the quark fields when

\footnotetext{
ae-mail: ganl@uncw.edu

${ }^{\mathrm{b}}$ This project is supported by USA NSF MRI PHY-0079840 and grant PHY-1206043.
}

This is an Open Access article distributed under the terms of the Creative Commons Attribution License 4.0, which permits unrestricted use, distribution, and reproduction in any medium, provided the original work is properly cited. 


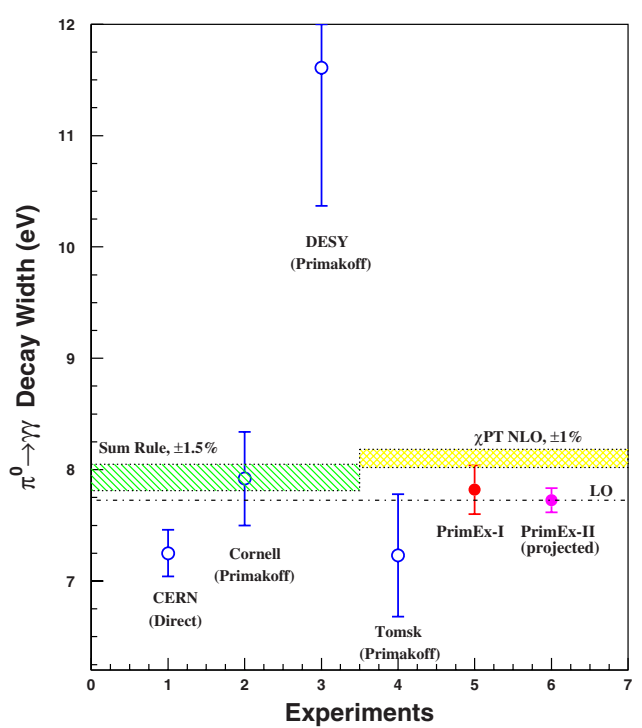

Figure 1. The $\pi^{0} \rightarrow \gamma \gamma$ decay width in $\mathrm{eV}$. The dashed horizontal line is the leading order prediction of the axial anomaly (Eq. (1) [3]). The left hand side shaded band is the recent QCD sum rule prediction [8] and the right hand side shaded band is the next-to-leading order chiral theory predictions $[5,6]$. The experimental results with errors are for: (1) the direct method [10]; $(2,3,4)$ the Primakoff method [11-13]; (5) published PrimEx-I result [1]; (6) the expected error for the PrimEx-II experiment, arbitrarily plotted to agree with the leading order prediction.

they couple to a gauge field. This phenomenon, called anomalous breaking [3], is of a pure quantum mechanical origin and can be calculated exactly to all orders. The predicted anomalous $\pi^{0} \rightarrow \gamma \gamma$ decay width $[3,4]$ is found by expanding the QCD chiral Lagrangian to first order in the pion field, yielding

$$
\Gamma\left(\pi^{0} \rightarrow \gamma \gamma\right)=\left(m_{\pi^{0}} / 4 \pi\right)^{3} /\left(\alpha / f_{\pi}\right)^{2}=7.76 \mathrm{eV}
$$

which has no adjustable parameters. The decay width given above is exact in the chiral limit. Since the mass of the $\pi^{0}$ is the smallest in the hadron spectrum, high order corrections to this prediction due to the non-vanishing quark masses are small $(\sim 4.5 \%)$ and can be calculated with sub-percent accuracy. Stimulated by the PrimEx project, several independent theoretical calculations have been published in the past decade, and are shown in Fig. 1. These calculations are performed either in the framework of chiral perturbation theory (ChPT) up to $\mathcal{O}\left(p^{6}\right)$ (NLO) [5, 6] (NNLO corrections are considered in [7]) or based on QCD sum rules [8], and they are consistent with an estimated theoretical uncertainty of $\sim 1 \%$. This offers one of the very few unique opportunities to test confinement QCD precisely.

The existing experimental results (prior to PrimEx) quoted by the Particle Data Group [9] are shown in Fig. 1. These results have large dispersion and their average value is $7.84 \pm 0.56 \mathrm{eV}$ with a $7 \%$ error, which is below the recent higher order theoretical predictions. In addition, the most precise measurement of the $\pi^{0}$ decay width, prior to the PrimEx experiment, used the direct method of measuring the mean decay length of the $\pi^{0}[10]$. Their result with the quoted $3.1 \%$ total uncertainty is $\sim 4 \sigma$ lower than the ChPT predictions [5-7]. The other three data points shown in Fig. 1 were done by the Primakoff method; however, they have large individual uncertainties and are inconsistent. This has given a strong motivation to the PrimEx collaboration to perform a new generation of Primakoff measurements of $\Gamma\left(\pi^{0} \rightarrow \gamma \gamma\right)$ at the $\simeq 1.4 \%$ accuracy to fill this important experimental gap.

The PrimEx experiment measures the $\pi^{0} \rightarrow \gamma \gamma$ decay width via small angle coherent photoproduction of the $\pi^{0}$ in the Coulomb field of a nucleus, i.e. the Primakoff effect. The production of $\pi^{0}$ 's in the Coulomb field of a nucleus by real photons is essentially the inverse of the decay $\pi^{0} \rightarrow \gamma \gamma$, and the cross section for this process thus provides a measure of the $\pi^{0}$ radiative decay width. The Primakoff cross section is peaked at small forward angle and strongly beam energy dependent $\left(d \sigma_{\operatorname{Pr}} / d \Omega \sim\right.$ $Z^{2} E^{4} / \mathrm{m}^{3}$ ). Consequently, it requires good angular resolution for the pion production angle in the forward direction, and high precision measurements of the energy and the rate of incident photons. Based on these requirements, our experiment was optimized to make a significant improvement over the 
previous Primakoff experiments [11-13]. The invariant mass and angle of the pion were reconstructed by detecting two decay photons from $\pi^{0}$ with a high resolution, high granularity $\mathrm{PbWO}_{4}$ calorimeter (HYCAL). A $\sim 5 \mathrm{GeV}$ photon beam was tagged by a high resolution photon tagger in Hall $\mathrm{B}$ to provide measurement of the energy and rate of incident photons. A pair production luminosity monitor was used for photon flux control during the production. The overall systematic uncertainty on the absolute cross section was verified by dedicated measurements of two QED processes: the electron Compton scattering and $e^{+} e^{-}$pair production with the same experimental apparatus; it was demonstrated to be better than $1.3 \%$ from the PrimEx-I result. There were two experiments performed on $\pi^{0}$. The first experiment (PrimEx-I) on two $5 \%$ radiation length (R.L.) targets of ${ }^{12} \mathrm{C}$ and ${ }^{208} \mathrm{~Pb}$ was performed in 2004. The result from PrimEx-I for the $\pi^{0}$ radiative decay width, $\Gamma\left(\pi^{0} \rightarrow \gamma \gamma\right)=7.82 \pm 0.14($ stat $) \pm 0.17($ sys $) \mathrm{eV}$ (shown in Fig. 1), was published in 2011 [1], with a total uncertainty of $2.8 \%$. In order to reach the ultimate goal of $\simeq 1.4 \%$ accuracy on the $\pi^{0}$ lifetime and to test QCD higher order corrections, the second experiment (PrimEx-II) was carried out on two $10 \%$ R.L. targets of ${ }^{12} \mathrm{C}$ and ${ }^{28} \mathrm{Si}$ in fall 2010 , with improved beam quality and charged particle rejection. The data analysis of PrimEx-II is currently in progress.

\section{Primakoff experiments at Jlab $12 \mathrm{GeV}$}

The $12 \mathrm{GeV}$ Primakoff experimental program [2] includes measurements of the two-photon decay widths of $\eta$ and $\eta^{\prime}$ and the transition form factors of $\pi^{0}, \eta$ and $\eta^{\prime}$ at $Q^{2}$ of $0.001-0.5 \mathrm{GeV}^{2} / \mathrm{c}^{2}$. While the former will require a real photon beam; the latter will be carried out through virtual Primakoff production with an electron beam. As described in the previous section, the $\pi^{0}$ decay offers the most sensitive probe to the chiral anomaly and spontaneous chiral symmetry breaking in QCD. On the other hand, due to larger masses, $\eta$ and $\eta^{\prime}$ decays are more sensitive to the breakings of SU(3) and isospin by the unequal quark masses. SU(3) breaking is primarily manifested by $\eta$ mixing with $\eta^{\prime}$, which contributes significantly in the next-to-leading order term in ChPT calculations. Precision measurement of $\eta$ and $\eta^{\prime}$ radiative decay widths will be critical input to determine the $\eta-\eta^{\prime}$ mixing angle. In addition, an accurate result for $\Gamma(\eta \rightarrow \gamma \gamma)$ will have a significant impact on the determination of the $m_{d}-m_{u}$ quark mass difference by improving the precision of the $\eta \rightarrow 3 \pi$ decay width [14]. The measurement of transition form factors of $\pi^{0}, \eta$ and $\eta^{\prime}$ at small $Q^{2}$ will model independently determine the electromagnetic interaction radii of those mesons. The measurements of $\eta^{\prime}$ will lead to a better understanding of the $\eta^{\prime}$ mass puzzle and its nature in general.

An experiment to measure $\Gamma(\eta \rightarrow \gamma \gamma)$ is currently under preparation at Jlab. The goal of this experiment is to measure the $\eta$ radiative decay width to the $3 \%$ level using the newly developed GlueX apparatus in Hall D. Due to a larger $\eta$ mass compared to $\pi^{0}$, the main challenges in the $\eta$ Primakoff experiment are: (1) smaller Primakoff cross section $\left(d \sigma_{P r} / d \Omega \sim Z^{2} E^{4} / m^{3}\right)$; (2) a larger overlap between the Primakoff signal and the nuclear hadronic background; (3) a larger momentum transfer to the target nucleus, which leads concerns about the coherency and form factors; and (4) more distortions from the in-medium effect. To address those issues, we will use a high energy tagged photon beam at $12 \mathrm{GeV}$ to increase the Primakoff cross section and to reduce the momentum transfer to the target. It will also increase the angular separation between the Primakoff signal $\left(<\theta_{P r}>_{\text {peak }} \sim m^{2} /\left(2 E^{2}\right)\right)$ and the nuclear coherent background $\left(<\theta_{N C}>_{\text {peak }} \sim 2 /\left(E \cdot A^{1 / 3}\right)\right)$. In addition, we choose two light targets: proton and ${ }^{4} \mathrm{He}$. While a proton target is free of the inelastic hadronic background and in-medium effect; the simplest compact nuclear target ${ }^{4} \mathrm{He}$ has a four times larger Primakoff cross section (compared to the proton), a well-known charge form factor, a minimal in-medium effect, and a full suppression of $\rho$-exchange in the nuclear coherent process.

The precise extraction of the Primakoff amplitude from the measured differential cross section requires good theoretical understanding of meson photoproduction mechanisms, including the processes of Primakoff, nuclear coherent and incoherent, and interference between Primakoff and 
nuclear coherent. In recent years, the light pseudoscalar meson photoproduction amplitudes in the electromagnetic and strong fields of nuclei at forward angles have been calculated independently by using the Glauber method [15] and Regge exchange [16]. In particular, the effects of initial and final state interactions in the nuclear medium, corrections for light nuclei, and contributions from nuclear collective excitations have been considered. Nuclear incoherent photoproduction has also been investigated using both Glauber theory [17] and the Cascade model [18]. Inspired by the PrimEx experiment, there have been two independent calculations of $\eta$ photoproduction on proton [19]. The virtual Primakoff production in high-energy electron-nucleus scattering has been calculated recently for the PrimEx kinematics [20]. These new theoretical developments have laid a solid theoretical ground for the era of precision Primakoff experiments for the light pseudoscalar mesons.

\section{Summary}

A comprehensive Primakoff experimental program has been developed by the PrimEx collaboration at Jlab to measure the two photon decay widths, $\Gamma_{\gamma \gamma}$, and the transition form factors, $F_{\gamma \gamma^{*}}$, of $\pi^{0}, \eta$, and $\eta^{\prime}$. It will provide a rich data set to test fundamental QCD symmetries at low energy.

\section{References}

[1] I. Larin et al. (PrimEx Collaboration), Phys. Rev. Lett. 106, 162303 (2011)

[2] Jefferson Lab $12 \mathrm{GeV}$ white paper, "The Science Driving the $12 \mathrm{GeV}$ Upgrade of CEBAF", February 2001

[3] J.S. Bell and R. Jaciw, Nuovo Cimento 60A, 47 (1969); S.L. Adler, Phys. Rev. 177, 2426 (1969)

[4] A.M. Bernstein and B.R. Holstein, Rev. Mod. Phys. 85, 49 (2013)

[5] J. L. Goity, A.M. Bernstein, B.R. Holstein, Phys. Rev. D 66, 076014 (2002)

[6] B. Ananthanarayan and B. Moussallam, J. High Energy Phys. 05, 052 (2002)

[7] K. Kampf and B. Moussallam, Phys. Rev. D 79, 076005 (2009)

[8] B. L. Ioffe and A. G. Oganesian, Phys. Lett. B 647, 389-393 (2007)

[9] C. Amsler et al. (Particle Data Group), PL B 667, 1 (2008)

[10] H.W. Atherton et al., Phys. Lett. 158B, 81 (1985)

[11] A. Browman et al., Phys. Rev. Letts. 33, 1400 (1974)

[12] G. Bellettini et al., Il Nuova Cimento 66, 243 (1970)

[13] V.I. Kryshkin et al., Sov. Phys. JETP 30, 1037 (1970)

[14] H. Leutwyler, Phys. Lett. B 374, 181 (1996); J. Bijnens and J. Gasser, Proceeding for the workshop on eta physics, Uppasala, October 25-27, 2001

[15] S. Gevorkyan, et al., Phys. Rev. C 80, 055201 (2009)

[16] Murat M. Kaskulov and Ulrich Mosel, Phys. Rev. C 84, 065206 (2011)

[17] S. Gevorkyan, et al., Phys. Part. Nucl. Lett. 9, 3 (2012)

[18] T.E. Rodrigues, et. al., Phys. Rev. Lett. 101, 012301 (2008)

[19] J.M. Laget, Phys. Rev., C 72, 022202 (2005); A. Sibirtsev, et al., Eur. Phys. J. A 44, 169 (2010)

[20] G. Faldt, Phys. Rev. C 83, 044606 (2011) and Phys. Rev. C 86, 024611 (2012) 